\title{
Phylogenetic Analysis of Mandarin Landraces, Wild Mandarins, and Related Species in China Using Nuclear LEAFY Second Intron and Plastid trnL-trnF Sequence
}

\author{
Li Yingzhi ${ }^{1}$, Cheng Yunjiang, Tao Nengguo, and Deng Xiuxin ${ }^{2}$ \\ National Key Laboratory of Crop Genetic Improvement, Huazhong Agriculture University, \\ Wuhan, Hubei Province, 430070, P. R. China
}

\begin{abstract}
AdDitional INDEX wORDS. Citrus reticulata, maximum parsimony, maximum likelihood, incongruence
Abstract. Nucleotide sequences of the second intron of the nuclear LEAFY gene (FLint2) and trnL-trnF region of the chloroplast genome were used to analyze the phylogenetic relationships among eight wild mandarins (Citrus reticulata Blanco), 19 mandarin landraces, and 19 related species of Citrus L. Forty-six trnL-trnF sequences and 111 FLint2 sequences were obtained from 46 ingroup accessions, with an average length of 1059.7 and 776.7 bp respectively. Phylogeny reconstructions were conducted separately for these two data sets using maximum parsimony and maximum likelihood criteria. Monophyly of mandarins was supported by both of these data sets, and in this clade, most mandarin landraces formed an unresolved polytomy, whereas 'Jiangyong 1', 'Chongyi A2', 'Chongyi A1' (or 'Jiangyong 4' in FLint2 data), and 'Daoxian 1' wild mandarins formed a subclade. 'Mangshan A1' and 'Daoxian 5' wild mandarins were sisters to this mandarin clade. A hybrid origin of five mandarin landraces and several mandarinrelated species was suggested as a plausible hypothesis to explain the incongruence between the FLint 2 and $\operatorname{trn} \mathrm{L}$-trnF data sets.
\end{abstract}

Relationships among species of Citrus are complicated by several factors such as a high frequency of bud mutation, a long history of cultivation, nucellar embryony , and wide crosscompatibility among species (Moore, 2001). The genus Citrus itself has been described as consisting of 1 to 162 species. The most widely accepted classification systems are those of Swingle (1943)and Tanaka (1977), who recognized 16 and 162 species respectively. A major difference in these two systems is in how mandarins (C. reticulata) are treated; 36 mandarin species are recognized by Tanaka $(1969,1977)$ whereas only three mandarin species are noted by Swingle's system (Swingle and Reece, 1967), including C. tachibana (Makino) Tanaka and C. indica Tanaka, wild species from Japan and India respectively. In the mid 1970s, two studies (Barrett and Rhodes, 1976; Scora, 1975) suggested that cultivated Citrus comprises only three basic species: citron (C. medica L.), mandarin (C. reticulata), and pummelo [C. maxima (Burm.) Merr.]. Later, C. halimii B.C. Stone was added as the fourth basic species (Scora, 1988). The status of these species was also supported by studies using biochemical and molecular markers (Fang and Roose, 1997; Fang et al., 1993; Herrero et al., 1996; Luro et al., 1992; Nicolosi et al., 2000).

Mandarins are the most phenotypically heterogeneous group in Citrus. Both monoembryonic and polyembryonic clones exist, as do self-fertile and self-incompatible types (Moore, 2001). High genetic similarity among members of some

Received for publication 30 Jan. 2007. Accepted for publication 10 July 2007. This research was supported by the National Natural Science Foundation of China (NSFC: no. 30500350 and 30471201); the International Foundation for Science (IFS, D/3814-1), Stockholm, Sweden; and IRT0548 of MOE, China. We thank Guo Wenwu for reviewing the manuscript.

${ }^{1}$ Current address: Horticultural and Forestry Department, Guangdong Ocean University, Zhanjiang, Guangdong Province, 524088, P. R. China.

${ }^{2}$ Corresponding author. E-mail: xxdeng@mail.hzau.edu.cn. mandarin cultivar groups has been detected in several studies (Coletta Filho et al., 1998; Esen and Scora, 1977; Machado et al., 1996), and great heterogeneity has been revealed within this group as well (Fang et al., 1998; Luro et al., 1995; Nicolosi et al., 2000; Torres et al., 1978). The mandarin group has been proposed to be composed of several genetically different individuals and a great number of hybrids (Coletta Filho et al., 1998).

China is believed to be the center of origin for citrus, and many mandarin landraces and wild mandarins are distributed there. In our previous study, high polymorphism was detected among these mandarin landraces and wild mandarins at simple sequence repeat (SSR) loci of both the chloroplast and nuclear genomes ( $\mathrm{Li}$ et al., 2006). These genetic resources provide a good opportunity to study the origin and phylogenetic relationship of mandarins.

Molecular markers offer a way to solve many of the longstanding problems in the classification and breeding of Citrus (Moore, 2001). DNA sequences of the chloroplast genome are widely used in the field of molecular plant systematics. The $t r n \mathrm{~L}$ intron and $\operatorname{trn} \mathrm{L}-\operatorname{trn} \mathrm{F}$ intergenic spacer exhibit a higher level of sequence variation among closely related species than the coding region, and vary in length and substitution rates (Clegg et al., 1994; Taberlet et al., 1991); therefore, they are more useful at lower taxonomic levels (Gielly and Taberlet, 1994). Recently, it was demonstrated that the $\operatorname{trn} \mathrm{L}-\operatorname{trn} \mathrm{F}$ intergenic spacer is informative and effective in determining phylogenetic relationships of Korean Citrus species (Jung et al., 2005). Several other regions of the chloroplast genome have been also used to elucidate the phylogenetic relationships of Rutaceae (Chase et al., 1999; Morton et al., 2003; Samuel et al., 2001; Scott et al., 2000). On the other hand, few nuclear DNA regions have been explored, with the exception of the nuclear ribosomal DNA region, which has been sequenced on a 
Table 1. Taxa and their sources used in the study of phylogenetic relationships of mandarin landraces, wild mandarin, and related species using trnL-F and LEAFY second intron (FLint2) sequences and number of FLint 2 clones sequenced.

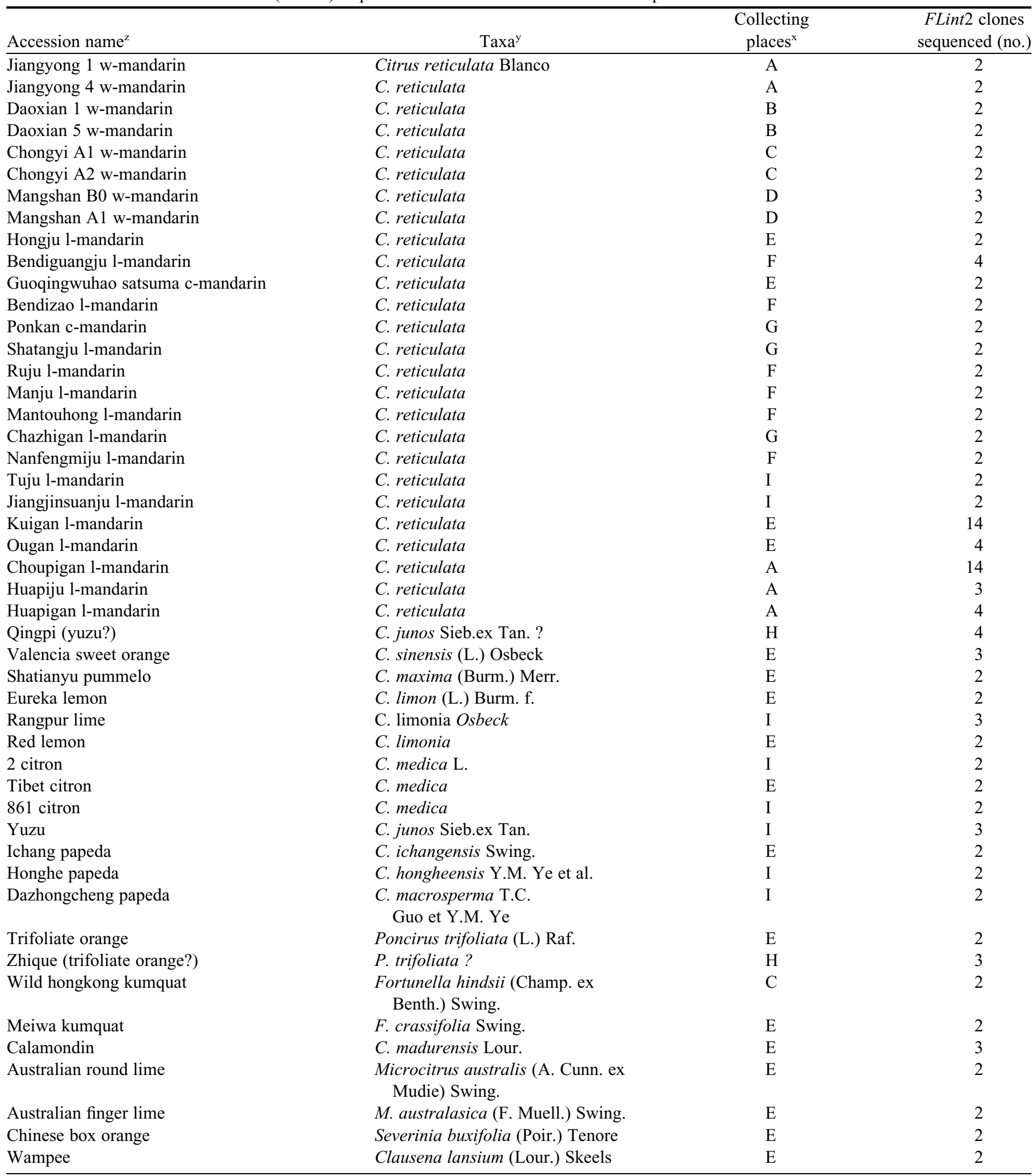

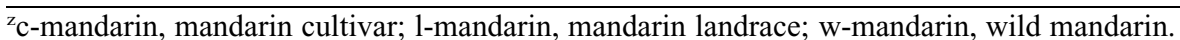

yTaxonomic treatment of mandarin followed Swingle's system (Swingle and Reece, 1967).

${ }^{x}$ A, Jiangyong, Hunan Province, China; B, Daoxian, Hunan Province, China; C, Chongyi, Jiangxi Province, China; D, Mangshan, Hunan Province, China; E, Citrus Research Institute of Huazhong Agricultural University, Wuhan, Hubei Province, China; F, Huangyan, Zhejiang Province, China; G, Sihui, Guangdong Province, China; H, Nanzhen, Shannxi Province, China; I, Citrus Research Institute of Chinese Academy of Agricultural Sciences, Chongqing, China. 
large scale. From this region, especially the internal transcribed spacers (ITS1 and ITS2) are extensively applied for phylogeny reconstruction at low taxonomic levels. However, ITS sequences are sometimes unsuitable for phylogenetic studies as a result of high sequence divergence (Wilson, 2003), extensive length variations between copies (Liston et al., 1996), paralogy problems (Baker et al., 2000), or lack of resolving power (Whitcher and Wen, 2001). FLORICAULA/LEAFY is a singlecopy nuclear-encoded homeotic gene in diploid angiosperms (Frohlich and Parker, 2000). There are three exons and two introns, and the locations of the two intros are well conserved (Frohlich and Meyerowitz, 1997). The utility of the second intron of $F L O / L F Y$ as a tool for phylogeny reconstruction at lower taxonomic levels has been documented in several studies, such as in Rosaceae (Oh and Potter, 2003) and Amorphophallus BI. ex Decne. (Grob et al., 2004).

In an effort to provide a molecular-based evolutionary tree of relationships among mandarin landraces and wild mandarins, we generated two new molecular data sets-trnL-trnF intergenic spacer of the chloroplast DNA (cpDNA), and $L E A F Y$ second intron (hereafter abbreviated as FLint2) of the nuclear DNA (nrDNA) - from 48 Citrus and relative accessions. The two data sets were analyzed to reconstruct independent phylogenetic trees with maximum likelihood and parsimony criteria to ascertain the robustness of their nodes.

\section{Material and Methods}

Plant materials and DNA extraction. Samples from 39 biotypes from seven species of Citrus, (among them, 18 were mandarin landraces and eight were wild mandarins) were collected. An additional seven accessions were also included to represent related genera: Fortunella Swingle, Poncirus Raf., and Microcitrus Swingle. Among mandarin accessions, Huapigan, Choupigan, Bendiguangju, Kuigan, and Ougan were proposed to be mandarin-associated hybrids in our previous study (Cheng et al., 2005; Li et al., 2006). Two taxa, wampee [Clausena lansium (Lour.) Skeels] and chinese box orange [Severinia buxifolia (Poir.) Ten.] were selected as outgroups following the results of the phylogenetic analyses of Rutaceae (Chase et al., 1999) and Aurantioideae (Morton et al., 2003), which suggested that Severinia Ten. and Clausena Burm. f. were sisters to Citrus. The collected accessions and their sources were given in Table 1. Number of FLint2 clones sequenced for each accession is also given in Table 1.

The collected leaves were immediately frozen in liquid nitrogen and stored at $-70{ }^{\circ} \mathrm{C}$ until DNA extraction. Total DNA was isolated from fresh young or mature leaves using a modified cetyltrimethyl ammonium bromide method (Cheng et al., 2003).

Polymerase chain reaction amplification and PURIFICATION OF ITS PRODUCTS. Two primers, LFY1 5' ATGC CGTGATTTCTTGATCC 3' and LFY2 5' TGGCATCAATA TCCCAACCT 3', were used in THE amplification of FLint2 for all accessions (N.G. Tao and X.X. Deng, unpublished). The chloroplast trnL-trnF region was amplified using $\mathrm{c}$ and $\mathrm{f}$ primers (Taberlet et al., 1991).

The target regions were amplified via polymerase chain reaction (PCR) in a Peltier Thermal Cycler-200 thermocycler (MJ Research, Watertown, MA). For each $\mathrm{PCR}$ reaction, $\approx 50$ ng total DNA was included in a $20-\mu \mathrm{L}$ reaction, consisting of $0.2 \mathrm{~mm}$ deoxynucleosides (dNTPs) (Shanghai Sangon Biolog- ical Engineering Technology and Services Corp., Shanghai, China), $0.1 \mu \mathrm{M}$ forward and reverse primers (Shanghai Sangon), $1 \times$ PCR buffer (Fermentas Canada, Burlington, ON, Canada), $1.5 \mathrm{~mm}$ magnesium chloride (Fermentas), and $1 \mathrm{U}$ Taq DNA polymerase (Fermentas). Thirty-five cycles of a three-step PCR followed by a final extension at $72{ }^{\circ} \mathrm{C}$ for $10 \mathrm{~min}$ were performed: denaturation at $94{ }^{\circ} \mathrm{C}$ for $1 \mathrm{~min}$, primer annealing at $55{ }^{\circ} \mathrm{C}$ for $40 \mathrm{~s}$, and primer extension at $72{ }^{\circ} \mathrm{C}$ for $1 \mathrm{~min}$.

Polymerase chain reaction products were separated on a $1.0 \%$ Tris-Acetate-EDTA (TAE)-agarose gel, cut out of the gel, and cleaned using E.Z.N.A. Gel Extraction Kit (Omega BioTek, Doraville, GA) following the manufacturer's protocols. The purified PCR products were cloned into the TaKaRa pMD18-T vector (TaKaRa Bio, Shiga, Japan), and inserts from one to four transformed colonies were sequenced with M13and $\mathrm{M} 13+$ primers.

All sequences were determined at Beijing SunBiotec Corp. (Beijing, China), which used an ABI 3770 automate sequencer (Applied BioSystems, Foster City, CA). Sequences were edited in Sequencher 4.1 (Genecodes Corp., Ann Arbor, MI).

SequenCE ALIGNMENTs. For FLint2, determined sequences were compared with $L E A F Y$ complete sequences of sweet orange [C. sinensis (L.) Osbeck, National Center for Biotechnology Information accession no. AY338976] and the exonintron boundaries were identified. Sequences were aligned using Clustal X (Thompson et al., 1997) and adjusted manually as needed. All sequences were deposited in GenBank databases, and aligned data matrices with the NEXUS file format (Maddison et al., 1997) were submitted to TreeBASE (Morell, 1996). These are available from the first author upon request.

Pairwise sequence divergence was calculated using Kimura's (1980) two-parameter (K2P) method in MEGA 3.0 (Kumar et al., 2004), with gaps/missing data completely deleted.

Outgroup Selection. The data sets were polarized by the outgroup method. For the trnL-trnF data set, wampee and chinese box orange were used as the outgroup. Outgroup comparisons with wampee were not amenable to use for phylogenetic comparison of FLnt 2 sequences because of significant sequence divergence; therefore, chinese box orange was used as the outgroup.

MAXimum PARSimony ANALYSIS. The maximum parsimony analyses were conducted using a heuristic search with tree bisection-reconnection (TBR) branch swapping, collapse of zero-length branches, and accelerated transformation. Characters were equally weighted; character states were specified as unordered. Random taxon addition (100 replicates) was used to search for multiple islands of trees. The strict consensus tree was generated based on the equally most-parsimonious trees produced by heuristic search. Bootstrapping analysis (Felsenstein, 1985) using heuristic search with random addition on 1000 bootstrap replicates was performed to determine relative support for various clades found in the parsimony analysis. All analyses were performed using PAUP* $4.0 \mathrm{~b} 10$ for Windows (Swofford, 2000).

In maximum parsimony analysis, we first treated gaps as missing data, and then treated them as fifth state to determine whether there were any phylogenetic topology differences between the two methods.

MAXIMUM LIKELIHOOD ANALYSIS. In all maximum likelihood analyses, heuristic searches with 500 replicates of random taxon addition, TBR branch swapping, and the MulTrees option were used. The best-fitting evolutionary model for each data in 
the maximum likelihood analysis was determined by the akaike information criterion using Modeltest 3.7 (Posada and Crandall, 1998). Gaps were treated as missing data and were excluded from analysis in maximum likelihood analysis.

Kishino-Hasegawa test and Shimodaira-Hasegawa TEST. To test which tree was best supported by sequence data sets, we used the Kishino-Hasegawa test (KH test) and the Shimodaira-Hasegawa test (SH test) as implemented in PAUP* $4.0 \mathrm{~b} 10$ (Swofford, 2000), with a model for each of the two data sets estimated using Modeltest 3.7 (Posada and Crandall, 1998), the reestimated log likelihoods method, and 1000 bootstrap replicates to compare different trees.
(Huapigan, Choupigan, Bendiguangju, Kuigan, and Ougan), wild mandarins, except two wild mandarins (Mangshan A1 and Daoxian 5), rangpur lime (C. limonia Osbeck) and red lemon (C. limonia), shared a 6-bp insertion in the same site. Kumquat (Fortunella sp.) biotypes, citron ( $C$. medica) biotypes, australian round lime (M. australis), and australian finger lime $[M$. australasica (F. Muell.) Swing.] shared a 6-bp deletion. Honghe papeda (C. hongheensis Y.M. Ye et al.) had a 5-bp insertion. None of these indels were parsimony informative.

After exclusion of indels, the matrix contained $921 \mathrm{bp}$, including 94 variable sites and 17 parsimony-informative sites. The $\operatorname{trn} \mathrm{L}-\operatorname{trn} \mathrm{F}$ data set used to study the phylogeny of Korean

\section{Results}

VARIATIONS AMONG TRNL-TRNF SEQUENCES. Forty-eight sequences were obtained in this study. The $\operatorname{trn} \mathrm{L}$ intron and $t r n \mathrm{~L}-t r n \mathrm{~F}$ intergenic spacer of Citrus and its relatives ranged in size from 1009 bp [dazhongcheng papeda ( $C$. macrosperma T.C. Guo et Y.M. Ye)] to 1083 bp (Tibet citron), with an average of $1059.7 \mathrm{bp}$. Nucleotide compositions were T, 28.8\%; C, $19.3 \%$; A, 33.6\%; and G, 18.3\%; and there were no differences in nucleotide compositions between mandarin landraces and wild populations.

Pairwise divergence between sequences was 0.00 to 0.018 in ingroup, and was 0.11 to 0.025 between outgroup and ingroup, with an average of 0.006 . Other study showed that the greatest sequence divergence among Korean citrus was between sour orange $(C$. aurantium L.) and C. natsudaidai Hay. (0.032) (Jung et al., 2005), different germplasm and different sequence length used in this study resulted in difference in sequence divergences. The transition-to-transversion ratio was 0.8 .

The aligned sequences, with introduced gaps, had a length of 1157 bp. The matrix contained 827 constant sites, 77 automorphic sites, and 17 parsimony-informative sites for the 48 accessions. Postulated insertions/deletions ranged from 1 to $27 \mathrm{bp}$ in length. Mangshan A1 wild mandarin had a 5-bp insertion, australian round lime [Microcitrus australis (A. Cunn. ex Mudie) Swingle] had a 27-bp insertion, and Fortunella sp. had a 27-bp insertion. All mandarin landraces, except possible mandarin-related hybrids

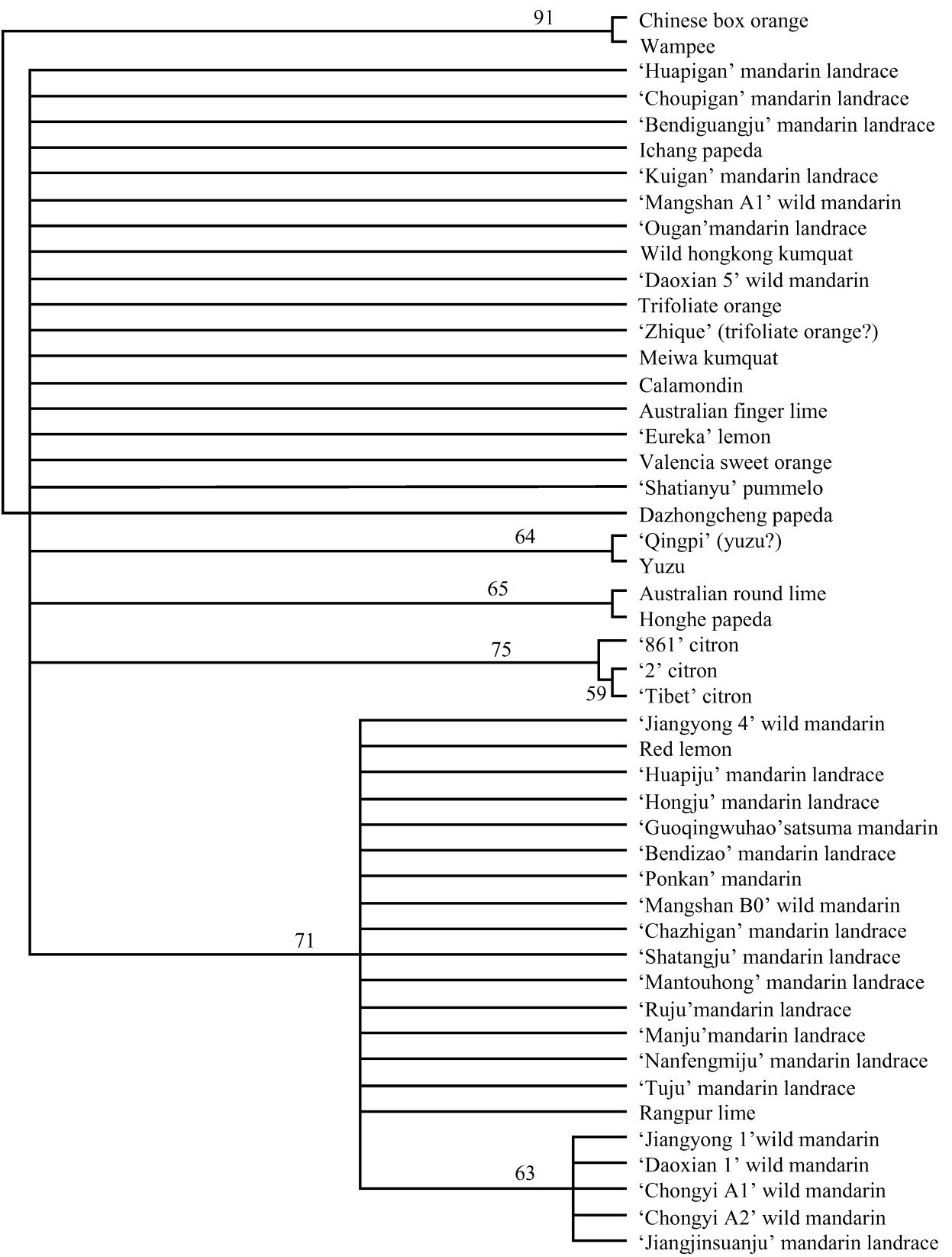

Fig. 1. Strict consensus tree (consistency index, 0.876 ; retention index, 0.794 ; tree length, 113 steps) of 16 equally most-parsimonious trees from the analysis of $\operatorname{trn} \mathrm{L}-\operatorname{trn} \mathrm{F}$ sequences in mandarins and related Citrus species. Bootstrap values (bootstrap percentage, $>50 \%$ ) of 1000 replications were shown around each branch. Gaps were excluded from the analysis. 
citrus was $372 \mathrm{bp}$ in length, with 67-bp variable sites (Jung et al., 2005). Again, a different germplasm and different sequence length used in these two studies resulted in a difference in sequence divergences.

SEQUenCe VARIATIONS AMONG LEAFY SECOND INTRON (FLINT2). Polymerase chain reaction amplification with LFY1 and LFY2 primers produced a single band in all accessions. For each accession, we sequenced more than two clones following the study of phylogenetic utility of FLint 2 in Neillia D. Don and Stephanandra Sieb. et Zucc. (Oh and Potter (2003). As a result, we obtained 135 sequences from 48 biotypes. The length of sequences of FLint 2 examined in this study varied from 756 to $783 \mathrm{bp}$, with an average of $776.7 \mathrm{bp}$. This variation was incited by one poly $\mathrm{T}$ tract and several indels in this region. The average nucleotide compositions were A, 26.7\%; T, 30.4\%; G, $18.3 \%$; and $\mathrm{C}, 24.6 \%$; and there were also no differences between mandarin landrace populations and wild mandarins.

Pairwise sequence divergence of $\mathrm{K} 2 \mathrm{P}$ distance ranged from 0.00 to 0.026 among the ingroup species, and from 0.023 to 0.035 between the ingroup taxa and the outgroup taxa, with an average of 0.012 . The transition-to-transversion ratio was 1.0 .

Nucleotide sequences of FLint2 from wampee were highly divergent from other samples; therefore, they were excluded from phylogenetic analysis. Because no significant differences were detected between different clones, only one clone per sample was included in the reduced data set. The final alignment of this reduced data set included 795 characters, of which 36 were phylogenetically informative and 91 were variable. Two insertions and one deletion had to be assumed to align sequences from chinese box orange and australian finger lime respectively.

After exclusion of indels, the data matrix was 753 bp in length, of which 91 sites were variable and 36 sites were parsimony informative.

Phylogenetic analysis using TRNL-TRNF DATA SET. We used two data sets for maximum parsimony analysis based on cpDNA sequences. First, the data set of 921 characters, excluding all indels, was analyzed. We obtained 16 equally most-parsimonious trees of 104 steps, a consistency index (CI) of 0.952 and retention index (RI) of 0.926. The strict consensus tree is shown in Fig. 1, with a tree length of 113 steps, a CI of 0.876 , and an RI of 0.794 .

Second, we added all indels and scored them as a fifth character. Phylogenetic analysis using 1157 characters yielded eight most-parsimonious trees of 515 steps with a CI of 0.680 and an RI of 0.682. A strict consensus tree (CI, 0.655; RI, 0.645; tree length, 534 steps) is shown in Fig. 2, and had more structures and increased branch resolution than the indelsexcluded parsimony tree.

The most appropriate model for the $\operatorname{trn} \mathrm{L}-\operatorname{trn} \mathrm{F}$ data determined in Modeltest was the TVM $+\mathrm{G}$ model with a shape parameter of gamma distribution, $\alpha=0.4240$, and a substitution matrix of six models. The best tree (-In $L=2595.20961)$ in the maximum

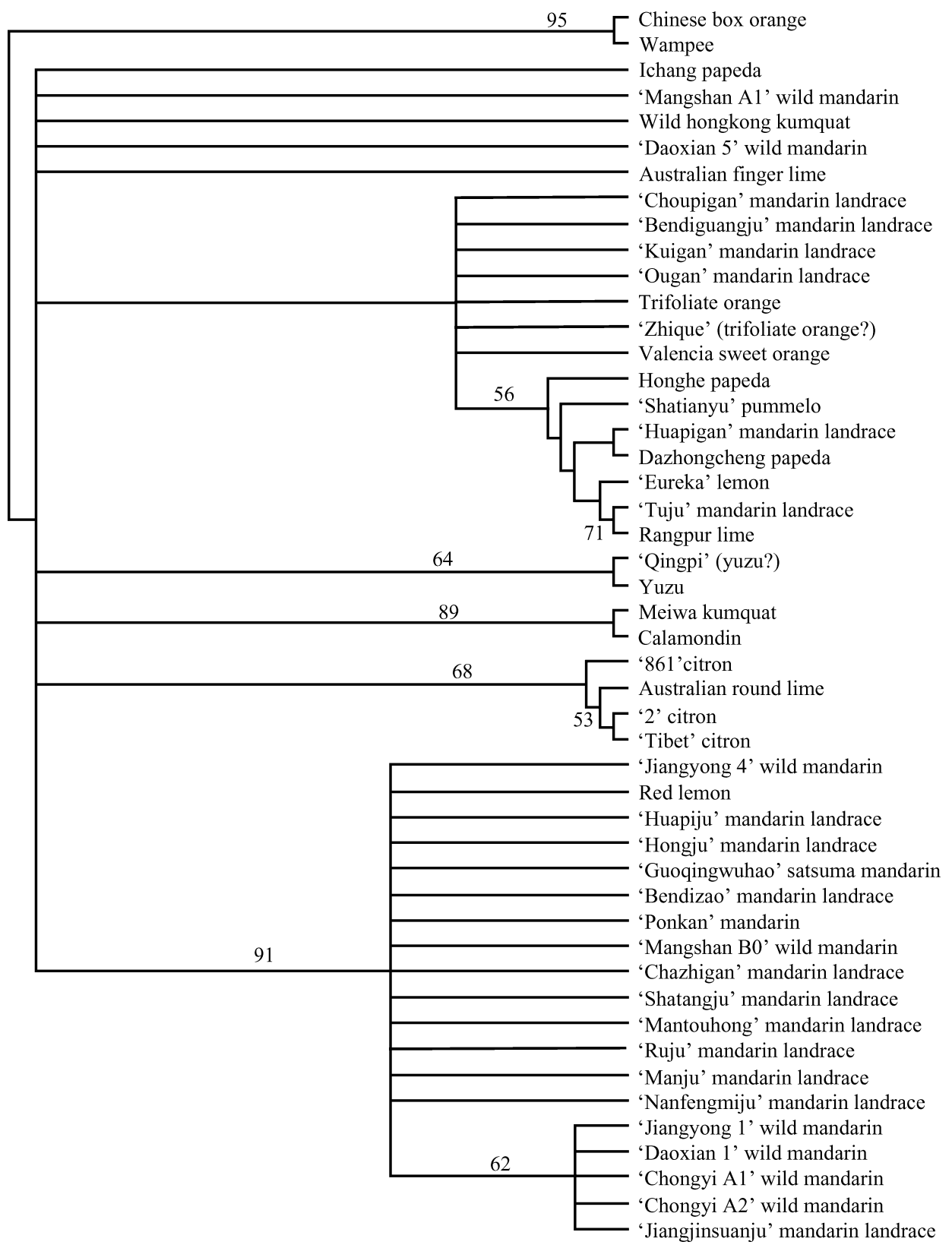

Fig. 2. Strict consensus tree (consistency index, 0.655; retention index, 0.645; tree length, 534 steps) of eight equally most-parsimonious trees from the analysis of $\operatorname{trn} \mathrm{L}-\operatorname{trn} \mathrm{F}$ sequences in mandarins and related Citrus species. Bootstrap values (bootstrap percentage, $>50 \%$ ) of 1000 replications were shown around each branch. Gaps were scored as fifth base. 


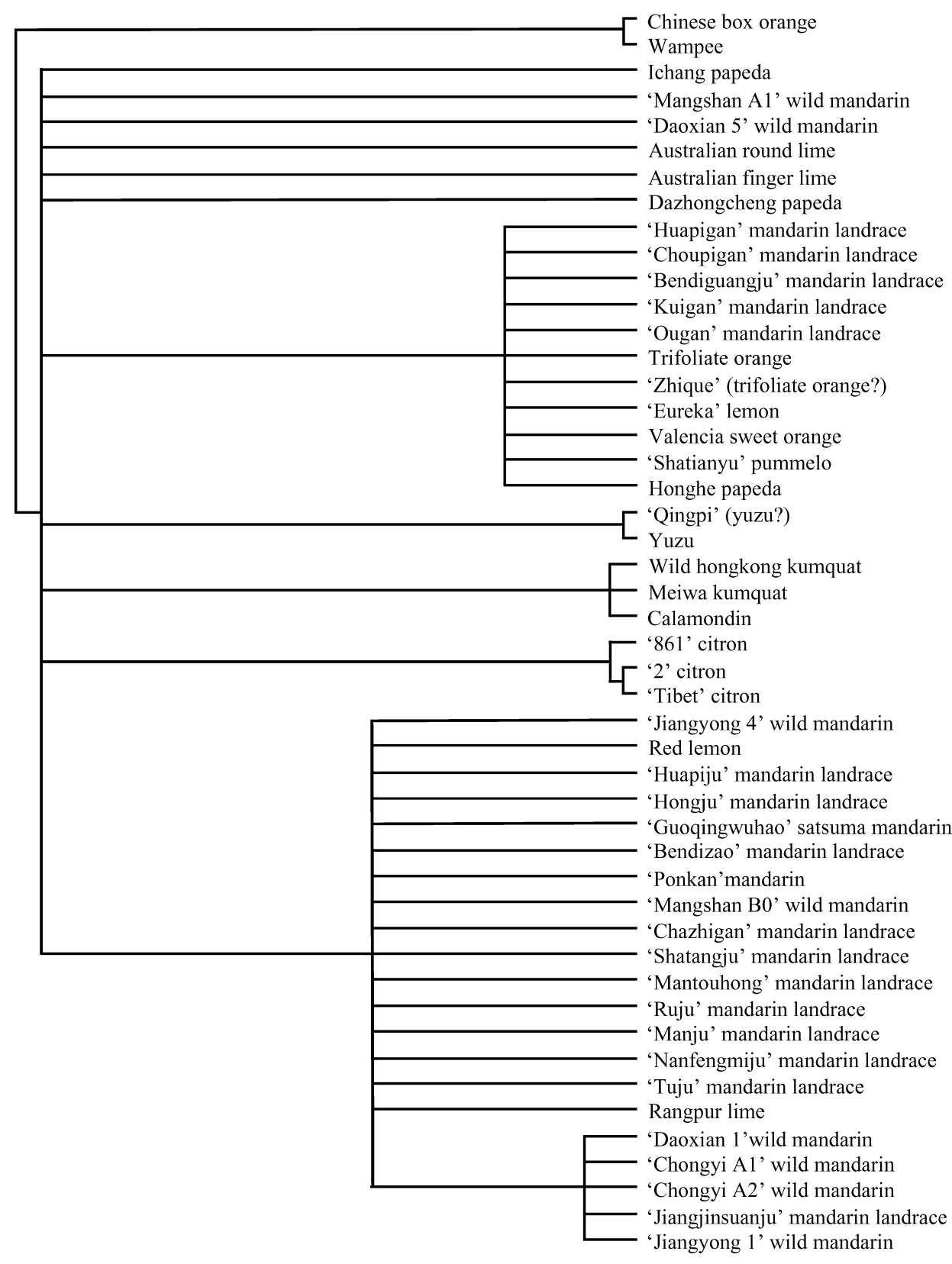

Fig. 3. Maximum likelihood reconstruction ( $-\mathrm{In} L=2595.20961)$ under the $\mathrm{TVM}+\mathrm{G}$ model for $\operatorname{trn} \mathrm{L}-\operatorname{trn} \mathrm{F}$ sequences in mandarins and related Citrus species. The best-fitting model was selected using Modeltest 3.7 (Posada and Crandall, 1998).

likelihood analysis (Fig. 3) using the model showed a few topology differences from the two strict consensus maximum parsimony trees.

The maximum likelihood tree was the best tree in the $\mathrm{KH}$ tests and the SH test, and the 16 indel information-excluded most-parsimonious trees were not different significantly from the maximum likelihood tree, whereas the eight indel information-included most-parsimonious trees were rejected for significantly different $(P<0.05)$.

All mandarin landraces, except five possible hybrid biotypes (Choupigan, Bendiguangju, Kuigan, Ougan, and Huapigan), formed a moderately supported monophyletic clade (hereafter called the mandarin clade I) in both parsimony [Figs. 1 and 2; bootstrap percentage (BP) $71 \%$ to $91 \%$ ] and likelihood (Fig. 3) trees. An exception was found for the Tuju mandarin landrace, which was placed outside this mandarin clade in the indel informationexcluded parsimony tree (Fig. 2). Five possible hybrid-origin mandarin landraces-Choupigan, Bendiguangju, Kuigan, Ougan, and Huapigan-were in a clade with sweet orange (C. sinensis), trifoliate orange [Poncirus trifoliata (L.) Raf.], pummelo, honghe papeda, and Eureka lemon [C. limon (L.) Burm. f.] in the indel informationexcluded parsimony consensus tree (no $>50 \%$ bootstrap value supported) and maximum likelihood tree, indicating that these mandarin biotypes had a closer relationship with pummelo and sweet orange.

Wild mandarins had the same topology in phylogenetic trees constructed with all three methods. All the wild mandarins, with the exception of Daoxian 5 and Mangshan A1, were nested within the mandarin clade. Jiangyong 1, Daoxian 1, Chongyi A1, and Chongyi A2 wild mandarins were loosely associated with Jiangjinsuanju mandarin landrace (BP, 62\% to $63 \%$ ); Mangshan B0 wild mandarin and Jiangyong 4 wild mandarins were sisters to these four wild mandarins (BP, $71 \%$ to $91 \%$ ). Daoxian 5 wild mandarin and Mangshan A1 wild mandarin were sisters to the mandarin clade I.

Rangpur lime and red lemon were contained in the mandarin clade $I$ in all parsimony and likelihood analyses of the $\operatorname{trn} \mathrm{L}-\operatorname{trn} \mathrm{F}$ sequence (BP, $71 \%$ to $91 \%$ ), indicating a closer relationship of these biotypes with mandarins in the chloroplast genome.

Qingpi (yuzu?) and yuzu (Citrus junos Siebold ex Tanaka) formed a weakly supported (BP, 63\% to 64\%) clade in the phylogenetic trees constructed by all three methods, showing a close relationship between them. Citron formed a monophyletic clade in all parsimony and likelihood trees (BP, 68\% to $75 \%$ ), and 2 citron and Tibet citron formed a subclade (BP, $53 \%$ to $59 \%$ ). Results of parsimony analysis with indel information included showed a sister relationship of australian round lime to the subclade of 2 citron/Tibet citron, but this was weakly supported (Fig. 2, BP, 53\%).

In the indel information-excluded parsimony tree (Fig. 1), relationships of meiwa kumquat (Fortunella crassifolia Swing.), wild hongkong kumquat [F. hindsii (Champ. ex Benth.) Swing.], and calamondin (C. madurensis Lour.) were unresolved and could not form a clade. However, in the 
parsimony tree with indels treated as a fifth character, meiwa kumquat and calamondin branched together with a bootstrap support of $89 \%$ (Fig. 2); and, in the maximum likelihood tree (Fig. 3), wild hongkong kumquat, meiwa kumquat, and calamondin formed an unresolved polytomy clade.

Phylogenetic analysis using data set Flint2. We used two data sets for maximum parsimony analysis based on Flint 2 sequences. First, all indels were excluded. A heuristic search found 12 equally most-parsimonious trees of 104 steps, a CI of 0.904 , and an RI of 0.940 . The $50 \%$ major-rule consensus tree and bootstrap values are shown in Fig. 4, with a tree length of 111 steps, a CI of 0.847 , and an RI of 0.898 .

Second, we added all indels and treated them as the fifth character. Phylogenetic analysis yielded 348 most-parsimoni- ous trees of 177 steps with a CI of 0.797 and an RI of 0.870 . A $50 \%$ major-rule consensus tree $(\mathrm{CI}, 0.750$; RI, 0.830; tree length, 188 steps) is shown in Fig. 5, and a few differences in topology and decreased branch resolution were found between this tree and that obtained by the first analyses.

The best evolutionary model for the FLint 2 data determined in Modeltest was $\mathrm{HKY}+\mathrm{G}$ with the shape parameter of gamma distribution, $\alpha=0.5279$, and a transition-to-transversion ratio of 1.3502. The best tree ( $-\operatorname{In} L=1935.55849)$ in the maximum likelihood analysis (Fig. 6) using the model showed the same topology as the strict consensus maximum parsimony tree derived from the indel information-excluded data matrix.

The $\mathrm{KH}$ test and the $\mathrm{SH}$ test found the maximum likelihood tree to be the best tree, and 12 indel information-excluded mostparsimonious trees and several indel information-included most-parsimonious trees were not different significantly from the maximum likelihood tree.

The mandarin landraces clustered as a large group (BP, 56\%), with two possible mandarin related hybrids (Kuigan and Choupigan) falling outside this clade (hereafter called the mandarin clade II). Both parsimony and likelihood analysis revealed that Choupigan mandarin landrace and Shatianyu pummelo together formed a strongly supported monophyletic group (BP, 83\% to $91 \%$ ), and that the Kuigan mandarin landrace is a sister to the Choupigan/Shatianyu pummelo clade supported by a low bootstrap value (BP, $57 \%$ or $<50 \%$ $\mathrm{BP})$. Tuju and Manju mandarin landraces formed a clade within this mandarin clade, with $61 \%$ to $67 \% \mathrm{BP}$ support, and likelihood and indelincluded parsimony analyses place Chongyi A1 wild mandarin as sister to this clade (Tuju/Manju), but no greater than $50 \%$ BP support was found in the parsimony tree.

All the wild mandarin accessions, except Mangshan A1 wild mandarin and Daoxian 5 wild mandarin, nested within the mandarin clade II. And, in this clade, Jiangyong 1 wild mandarin, Daoxian 1 wild mandarin, Jiangyong 4 wild mandarin, and Chongyi A2 wild mandarin branched together (BP, $52 \%$ to $66 \%$ ), with Jiangyong 1 being sister to other three biotypes (BP, $50 \%$ to $62 \%$ ). Mangshan A1 wild mandarin and Daoxian 5 wild mandarin were sisters to the mandarin clade II.

In this study, clustering of australian round lime (M. australis) with Mangshan A1 wild mandarin was the most distinct in the Flint2 data set analysis. In all phylogenetic trees
Fig. 4. Strict consensus tree (consistency index, 0.847; retention index, 0.898; tree length, 111 steps) of 12 equally most-parsimonious trees from the analysis of FLint 2 sequences in mandarins and related Citrus species. Bootstrap values (bootstrap percentage, $>50 \%$ ) of 1000 replications were shown around each branch. Gaps were excluded from the analysis. 


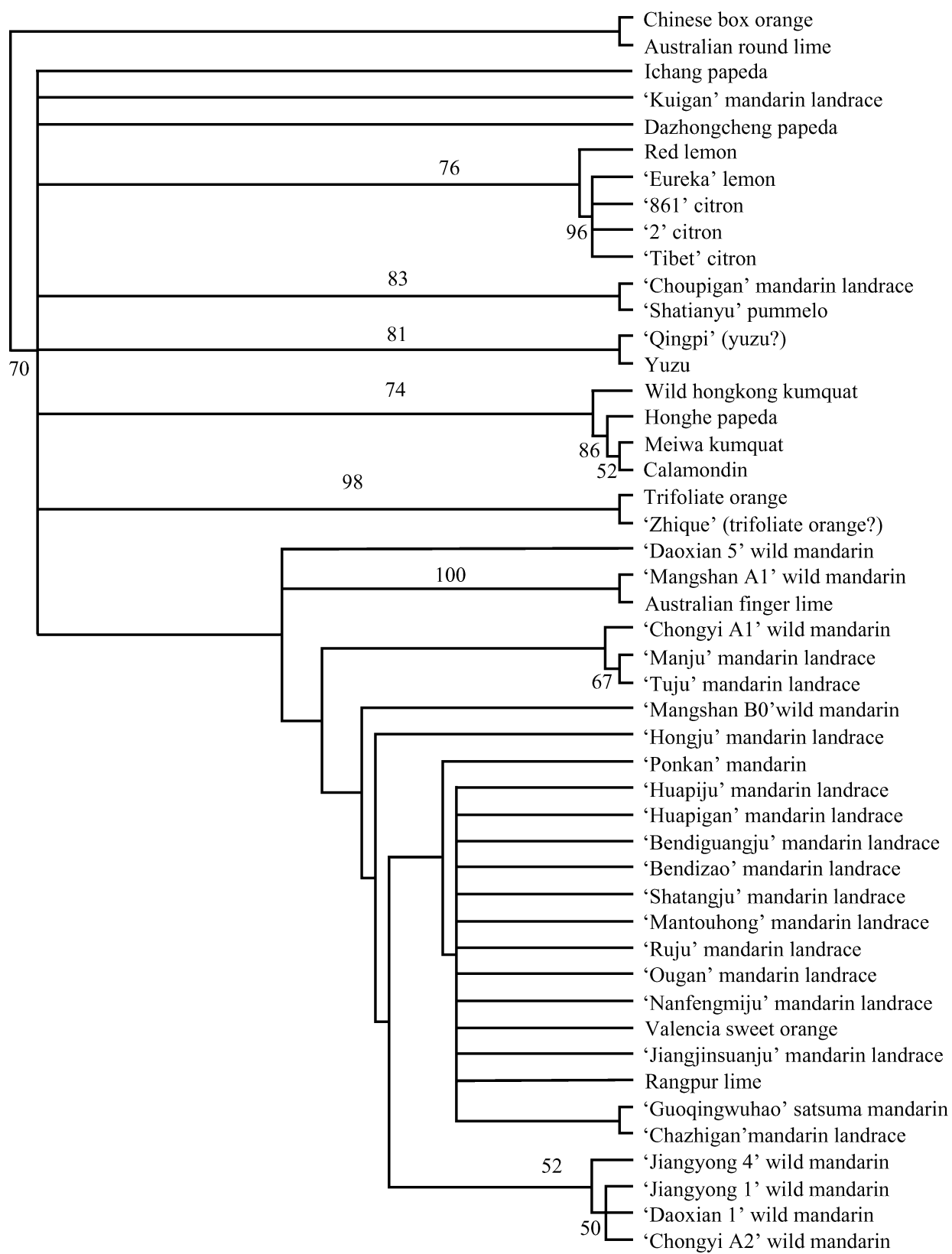

Fig. 5. Strict consensus tree (consistency index, 0.750; retention index, 0.830; tree length, 188 steps) of 348 equally most-parsimonious trees from the analysis of FLint 2 sequences in mandarins and related Citrus species. Bootstrap values (bootstrap percentage, $>50 \%$ ) of 1000 replications were shown around each branch. Gaps were treated as fifth base.

resulting from the FLint2 analysis, Mangshan A1 wild mandarin and australian round lime formed a highly supported clade (BP, 100\%). They are significantly different in morphology and geographical distribution, so this result is difficult to explain.

Results of indel-excluded parsimony and maximum likelihood analysis showed a sister-group relationship of ichang papeda (C. ichangensis Swing.) and dazhongcheng papeda (C. macrosperma) to the Qingpi/yuzu clade, but this is not well supported by parsimony analysis (BP, 62\%).

Rangpur lime and valencia sweet orange $(C$. sinensis $)$ rested among biotypes belonging to mandarin in parsimony and likelihood trees, and therefore were more related to mandarin than to other species.
Eureka lemon and all three biotypes of citron formed a strongly supported polytomy (BP, 96\% to $98 \%$ ), and red lemon is sister to this clade supported by a relatively high bootstrap value (BP, $73 \%$ to $76 \%$ ).

As in the $\operatorname{trn} \mathrm{L}-\operatorname{trn} \mathrm{F}$ data set, Qingpi and yuzu clustered together in all the maximum parsimony and maximum likelihood analyses of the FLint2 data set (BP, 63\% to $81 \%$ ), again showing a close relationship between these two accessions.

Honghe papeda, meiwa kumquat, and calamondin produced a wellsupported polytomy clade in both the parsimony (BP, $84 \%$ to $86 \%$ ) and likelihood trees, and only in the indel information-included parsimony tree, meiwa kumquat and calamondin could formed a weakly supported subclade (BP, 52\%). The position of wild hongkong kumquat as sister to these three biotypes was only moderately supported by indel information-included parsimony analysis (BP, 74\%).

Different from cpDNA analyses results, in which, the relationship between trifoliate orange and ' $\mathrm{Zhi}$ que' (trifoliate orange?) was unresolved, in nrDNA analyses results, these two accessions formed a highly supported clade in all maximum parsimony and maximum likelihood trees (BP, $97 \%$ to $98 \%$ ).

\section{Discussion}

RELATIONSHiPS AMONG WILD MA N D A R I N A N D MANDARIN LANDRACES. Both the phylogenetic analysis of nrDNA and cpDNA sequence data showed that wild mandarins were not a monophyletic group, and were divided into three groups.

Sister relationships of Mangshan A1 wild mandarin and Daoxian 5 wild mandarin to all the nonhybrid mandarin landraces were supported by both the cpDNA and nrDNA analysis. Jiangyong 1, Daoxian 1, and Chongyi A2 wild mandarins formed a subclade within the mandarin clade I and II in cpDNA (BP, $62 \%$ to $63 \%$ ) and nrDNA (BP, $52 \%$ to $66 \%$ ); however, cpDNA analysis placed Chongyi A1 wild mandarin in the mandarin clade I whereas nrDNA analysis placed Jiangyong 4 wild mandarin in the mandarin clade II. The other accessions of wild mandarin, Jiangyong 4 and Mangshan B0, Jiangyong 1, and Mangshan B0 were sisters to this wild mandarin subclade in cpDNA and nrDNA respectively.

A relatively loose association of Jiangjinsuanju mandarin landrace and wild mandarins (Jiangyong 1, Daoxian 1, Chongyi A1, Chongyi A2) was revealed by cpDNA analyses (BP, $62 \%$ to 


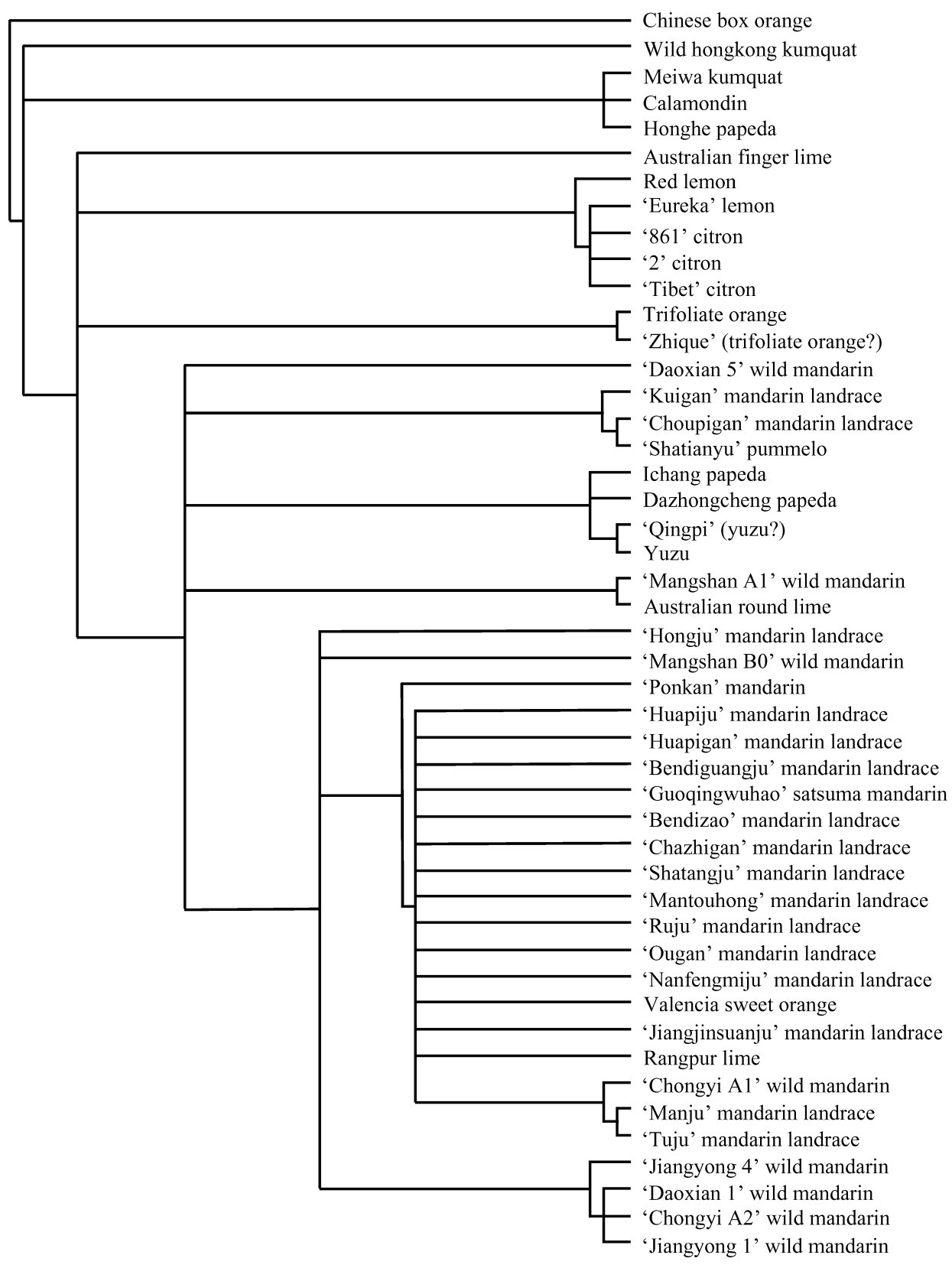

Fig. 6. Maximum likelihood reconstruction (-In $L=1935.55849$ ) of phylogeny under the HKY+G model for FLint 2 sequences in mandarins and related Citrus species. The best-fitting model was selected using Modeltest 3.7 (Posada and Crandall, 1998)

$63 \%$ ). Chongyi A1 wild mandarin appeared to be sister to the $\mathrm{Manju}$ /Tuju mandarin landrace clade in nrDNA analyses results (Figs. 5 and 6), but this was not well supported (BP, 67\%).

Relationships of Citrus and its Relatives. The most commonly invoked cause for incongruence among nuclear and plastid DNA markers is hybridization. Because of the widely accepted belief that most Citrus species have been derived from hybridization (Federici et al., 1998; Handa et al., 1986; Moore, 2001), we attempted to use the incongruence between a cpDNA data set and an nrDNA data set to decide which species are hybrids.

Red lemon was believed to be a hybrid between citron and mandarin (Fang et al., 1993). In this study, red lemon was contained within a clade including lemon $(C$. limon) and citron
(C. medica) in the analysis of the nrDNA data set, whereas it nested among mandarins in the analysis of the cpDNA data set. From this point of view, we propose that red lemon is a hybrid between mandarin and lemon or citron, with the former as the maternal parent and the latter as the male parent - a view consistent with previous suggestions that citron ( $C$. medica) is probably a parent of limes and lemons (Barrett and Rhodes, 1976; Federici et al., 1998; Malik et al., 1974; Scora, 1975).

A citron $\times$ mandarin hybrid ancestry was proposed for rangpur lime in a previous study (Nicolosi et al., 2000). However, we could not solve the relationship of rangpur lime to mandarin and citron in this analysis. It has a close association with mandarin in nrDNA data set analysis, but uncertainty in the cpDNA data set analysis. In indel information-excluded parsimony tree and maximum likelihood tree, it clustered with all nonhybrid mandarin landraces, but in indel information-included parsimony tree, it branched together with the Tuju mandarin landrace (BP, 71\%) as a part of a large clade including lemon, papeda, and pummelo (BP, 56\%).

Swingle (1943) suggested calamondin to be a hybrid between kumquat and mandarin, whereas some studies did not support kumquat as its parent (Torres et al., 1978), suggesting that Fortunella species were a single, independent group (Rahman and Nito, 1994), with a single maternal ancestor (Abkenar et al., 2004). Our results showed that, in both nrDNA analysis and cpDNA analysis, meiwa kumquat and calamondin formed a monophyletic clade, and a sister relationship of honghe papeda was moderately supported by nrDNA analysis. Another kumquat species in this study, wild hongkong kumquat, was sister to these two kumquat species, but this was weakly supported by both the nrDNA (Fig. 5; BP, 74\%) and cpDNA (Fig. 3). Despite the fact that the relationship of calamondin and kumquat was not resolved in this study, the relationship of calamondin with mandarin was not supported by our results, although sample errors (e.g., undetected sequences in cloning procedures) cannot be ruled out as possibilities.

Both the trnL-trnF data set and the Flint2 data set support Qingpi as being the same species as yuzu, and it was different significantly from mandarin landraces both in this study and in a previous study based on nuclear and chloroplast SSR data (Li et al., 2006). Besides, in Flint2 analysis, these two biotypes formed a clade with ichang papeda and dazhongcheng papeda. 
Previously, Swingle and Reece (1967) suggested that yuzu is a hybrid between ichang papeda and mandarin. Our results support the nuclear genome of yuzu coming from papeda, but the origin of their chloroplast genomes was not resolved in this study.

Another two biotypes found to be the same species were trifoliate orange and Zhique, despite divergence in their cpDNA sequences. These results were consistent with results from nuclear simple sequence and chloroplast simple sequence analysis (Y.Z. Li and X.X. Deng, unpublished).

Huapigan, Choupigan, Kuigan, Bendiguangju, and Ougan mandarin landraces have been proposed to be of hybrid origin, with pummelo/orange and mandarin being parents (Cheng et al., 2005; Li et al., 2006). This study further supported this hypothesis, because Huapigan, Bengdiguangju, and Ougan clustered with mandarin accessions in the analysis of nrDNA, whereas distributed in the pummelo/orange clade in the analysis of cpDNA indicating that mandarin was their male parent and pummelo/orange was their female parent. However, FLint2 analysis placed Kuigan, Choupigan, and pummelo in a clade. We proposed that only the maternal copy of the gene is present in these biotypes, and sampling of other low-copy genes may provide further information.

In this study, valencia sweet orange had a close association with mandarin in nrDNA, whereas it had a close association with pummelo in cpDNA, supporting the theory that sweet oranges are a hybrid between mandarin and pummelo (Barrett and Rhodes, 1976; Luro et al., 1995; Nicolosi et al., 2000), with mandarin as the male parent and pummelo as the female parent (Cheng et al., 2005).

\section{Literature Cited}

Abkenar, A.A., S. Isshiki, and Y. Tashiro. 2004. Phylogenetic relationships in the "true citrus fruit trees" revealed by PCR-RFLP analysis of cpDNA. Sci. Hort. 102:233-242.

Baker, W.J., T.A. Hedderson, and J. Dransfield. 2000. Molecular phylogenetics of subfamily Calamoideae (Palmae) based on nrDNA ITS and cpDNA rps 16 intron sequence data. Mol. Phylogenet. Evol. 14:195-217.

Barrett, H.C. and A.M. Rhodes. 1976. A numerical taxonomic study of affinity relationships in cultivated citrus and its close relatives. Syst. Bot. 1:105-136.

Chase, M.W., C.M. Morton, and J.A. Kallunki. 1999. Phylogenetic relationships of Rutaceae: A cladistic analysis of the subfamilies using evidence from $r b c \mathrm{~L}$ and $a t p \mathrm{~B}$ sequence variation. Amer. J. Bot. 86:1191-1199.

Cheng, Y.J., V.M. De Carmen, De, H.J. Meng, W.W. Guo, N.G. Tao, and X.X. Deng. 2005. A set of primers to analyze the chloroplast DNA diversity in Citrus and its relatives. Tree Physiol. 25:661-672.

Cheng, Y.J., W.W. Guo, H.L. Yi, X.M. Pang, and X.X. Deng. 2003. An efficient protocol for genomic DNA extraction from Citrus species. Plant Mol. Biol. Rptr. 21:177a-177g.

Clegg, M.T., B.S. Gaut, G.H. Learn, Jr., and B.R. Morton. 1994. Rates and patterns of chloroplast DNA evolution. Proc. Natl. Acad. Sci. USA 91:6759-6801.

Coletta Filho, H.D., M.A. Machado, M.L.P.N. Targon, M.C.P.Q.D.G. Moreira, and J. Pompeu, Jr. 1998. Analysis of the genetic diversity among mandarins (Citrus spp.) using RAPD markers. Euphytica 102:133-139.

Esen, A. and R.W. Scora. 1977. Analyses polymorphism in Citrus and some related genera. Amer. J. Bot. 64:305-309.

Fang, D.Q., R.R. Krueger, and M.L. Roose. 1998. Phylogenetic relationships among selected Citrus germplasm accessions revealed by inter-simple sequence repeat (ISSR) markers. J. Amer. Soc. Hort. Sci. 123:612-617.
Fang, D.Q. and M.L. Roose. 1997. Identification of closely related citrus cultivars with inter-simple sequence repeat markers. Theor. Appl. Genet. 95:408-417.

Fang, D.Q., W.C. Zhang, and S.Y. Xiao. 1993. Studies on taxonomy and evolution of Citrus and its related genera by isozyme analysis. Acta Phytotaxonomica Sinica 31:329-352 (In Chinese with English abstract).

Federici, C.T., D.Q. Fang, R.W. Scora, and M.L. Roose. 1998. Phylogenetic relationships within the genus Citrus (Rutaceae) and related genera as revealed by RFLP and RAPD analysis. Theor. Appl. Genet. 96:812-822.

Felsenstein, J. 1985. Confidence limits on phylogenies: An approach using the bootstrap. Evolution Int. J. Org. Evolution 39:783-791.

Frohlich, M.W. and E.M. Meyerowitz. 1997. The search for homeotic gene homologs in basal angiosperms and Gnetales: A potential new source of data on the evolutionary origin of flowers. Int. J. Plant Sci. 158:S131-S142.

Frohlich, M.W. and D.S. Parker. 2000. The mostly male theory of flower evolutionary origins: From genes to fossils. Syst. Bot. 25:155170.

Gielly, L. and P. Taberlet. 1994. Chloroplast DNA polymorphism at the intrageneric level: Implications for the establishment of plant phylogenies. Comptes Rendues de L'Acadamie des Sciences (Life Sci.) 317:685-692.

Grob, G.B.J., B. Gravendeel, and M.C.M. Eurlings. 2004. Potential phylogenetic utility of the nuclear FLORICAULA/LEAFY second intron: Comparison with three chloroplast DNA regions in Amorphophallus (Araceae). Mol. Phylogenet. Evol. 30:13-23.

Handa, T., Y. Ishizawa, and C. Oogaki. 1986. Phylogenic study of fraction I protein in the genus Citrus and its close related genera. Jpn. J. Genet. 61:15-24.

Herrero, R., M.J. Asins, E.A. Carbonell, and L. Navarro. 1996. Genetic diversity in the orange subfamily Aurantioideae. I. Intraspecies and intragenus genetic variability. Theor. Appl. Genet. 92:599-609.

Jung, Y.H., H.M. Kwon, S.H. Kang, J.H. Kang, and S.C. Kim. 2005. Investigation of the phylogenetic relationships within the genus Citrus (Rutaceae) and related species in Korea using plastid trnLtrnF sequences. Sci. Hort. 104:179-188.

Kimura, M. 1980. A simple method for estimating evolutionary rate of base substitutions through comparative studies of nucleotide sequences. J. Mol. Evol. 16:111-120.

Kumar, S., K. Tamura, and M. Nei. 2004. MEGA3: Integrated software for Molecular Evolutionary Genetics Analysis and sequence alignment. Brief. Bioinform. 5:150-163.

Li, Y.Z., Y.J. Cheng, H.L. Yi, and X.X. Deng. 2006. Genetic diversity in mandarin landraces and wild mandarins from China based on nuclear and chloroplast simple sequence repeat markers. J. Hort. Sci. Biotechnol. 81:371-378.

Liston, A., W.A. Robinson, J.M. Oliphant, and E.R. Alvarez-Buylla. 1996. Length variation in the nuclear ribosomal DNA internal transcribed spacer region of non-flowering seed plants. Syst. Bot. 21:109-120.

Luro, F., F. Laigret, and J.M. Bove. 1992. Application of random amplified polymorphic DNA (RAPD) to Citrus genetics and taxonomy. Proc. Intl. Soc. Citriculture 1:225-228.

Luro, F., F. Laigret, J.M. Bove, and P. Ollitrault. 1995. DNA amplified fingerprinting: A useful tool for determination of genetic origin and diversity analysis in Citrus. HortScience 30:1063-1067.

Machado, M.A., H.D. Colleta Filho, M.L.P.N. Targon, and J. Pomper, Jr. 1996. Genetic relationship of Mediterranean mandarins (Citrus deliciosa Tenore) using RAPD markers. Euphytica 92:321-326.

Maddison, D.R., D.L. Swofford, and W.P. Maddison. 1997. NEXUS: An extensible file format for systematic information. Syst. Biol. 46:590-621.

Malik, M.N., R.W. Scora, and P.K. Soost. 1974. Studies on the origin of the lemon. Hilgardia 42:361-382.

Moore, G.A. 2001. Oranges and lemons: Clues to the taxonomy of Citrus from molecular markers. Trends Genet. 17:536-540. 
Morell, V. 1996. TreeBASE: The roots of phylogeny. Science 273:569.

Morton, C.M., M. Grant, and S. Blackmore. 2003. Phylogenetic relationships of the Aurantioideae inferred from chloroplast DNA sequence data. Amer. J. Bot. 90:1470-1480.

Nicolosi, E., Z.N. Deng, A. Gentile, S. La Malfa, G. Continella, and E. Tribulato. 2000. Citrus phylogeny and genetic origin of important species as investigated by molecular markers. Theor. Appl. Genet. 100:1155-1166.

Oh, S. and D. Potter. 2003. Phylogenetic utility of the second intron of LEAFY in Neillia and Stephanandra (Rosaceae) and implications for the origin of Stephanandra. Mol. Phylogenet. Evol. 29:203215.

Posada, D. and K.A. Crandall. 1998. Modeltest: Testing the model of DNA substitution. Bioinformatics 14:817-818.

Rahman, M.M. and N. Nito. 1994. Phylogenetic relationships in the kumquat (Fortunella) as revealed by isozyme analysis. Sci. Hort. 57:17-28.

Samuel, R., F. Ehrendorfer, M.W. Chase, and H. Greger. 2001. Phylogenetic analyses of Aurantioideae (Rutaceae) based on noncoding plastid DNA sequences and phytochemical features. Plant Biol. 3:77-87.

Scora, R.W. 1975. On the history and origin of Citrus. Bul. Torrey Bot. Club 102:369-375.

Scora, R.W. 1988. Biochemistry, taxonomy and evolution of modern cultivated citrus. Proc. Intl. Soc. Citriculture 1:277-289.

Scott, K.D., C.L. McIntyre, and J. Playford. 2000. Molecular analyses suggest a need for a significant rearrangement of Rutaceae subfamilies and a minor reassessment of species relationships within Flindersia. Plant Syst. Evol. 223:15-27.
Swingle, W.T. 1943. The botany of Citrusand its wild relatives of the orange subfamily, p. 129-474. In: H.J. Webber and L.D. Batchelor (eds.). The citrus industry, vol. 1. University of California, Berkeley, CA.

Swingle, W.T. and P.C. Reece. 1967. The botany of Citrus and its wild relatives, p. 190-430. In: W. Reuther, H.J. Webber, and L.D. Batchelor (eds.). The citrus industry, vol. 1. University of California, Berkeley, CA.

Swofford, D.L. 2000. PAUP*. Phylogenetic analysis using parsimony (*and other methods). Version 4b. Sinauer Associates, Sunderland, MA.

Taberlet, P., L. Gielly, G. Pautou, and J. Bouvet. 1991. Universal primers for amplification of three non-coding regions of chloroplast DNA. Plant Mol. Biol. 17:1105-1109.

Tanaka, T. 1969. Misunderstanding with regard to citrus classification and nomenclature. Bul. Univ. Osaka Prefecture Ser. B 21:139-145.

Tanaka, T. 1977. Fundamental discussion of citrus classification. Study Citrologia 14:1-6.

Thompson, J.D., T.J. Gibson, F. Plewniak, F. Jeanmougin, and D.G. Higgins. 1997. The clustal-X windows interface: flexible strategies for multiple sequence alignment aided by quality analysis tools. Nucl. Acids Res. 25:4876-4882.

Torres, A.M., R.K. Soost, and U. Diedenhofen. 1978. Leaf isozymes as genetic markers in citrus. Amer. J. Bot. 65:869-881.

Whitcher, I.N. and J. Wen. 2001. Phylogeny and biogeography of Corylus (Betulaceae): Inferences from ITS sequences. Syst. Bot. 26:283-298.

Wilson, C.A. 2003. Phylogenetic relationships in Iris series Californicae based on ITS sequences of nuclear ribosomal DNA. Syst. Bot. 28:39-46. 